\title{
IL PESO DELLE OBBLIGAZIONI NELLA DOCUMENTAZIONE NOTARILE DEL CENTRO-NORD D'ITALIA
}

\author{
THE WEIGHT OF BONDS IN THE NOTARIAL DOCUMENTATION \\ OF CENTRAL-NORTHERN ITALY
}

\author{
Miriam Davide \\ Università di Trieste
}

\begin{abstract}
English: The essay examines the different types of bonds present in the documentation of Central-Northern Italy in the framework of regulation written in the notarial formularies of Ranieri di Perugia, Salatiele and Zaccaria Martino, where these acts follow the traditional documents like sales, donations and wills. Credit deeds are written in a similar way since the same standards are present everywhere with a sort of common plot. In most Italian towns, notaries usually write credit documents on vacchette, which contain the main information relating to the contract, directly in front of the interested parties. Then they transcribe the same acts in registers in which the extended version of the documents is proposed. The writing of the rough copy generally includes all guarantee clauses in a concise way; subsequent registration in the notarial protocols is authenticated by the notaries and guaranteed by the public authority. The use of notebooks of minuta is simultaneous with the increase of credit agreements and in their organization in the notarial formularies.
\end{abstract}

Keywords: Credit; Notarial Formularies; Middle Ages; Northern Italy; Moneylenders

Abstract Italiano: Il saggio prende in esame le diverse tipologie di contratti di obbligazioni presenti nella documentazione del Centro-Nord d'Italia nel quadro della normativa giuridica prodotta a partire dai formulari di Ranieri di Perugia, Salatiele e Zaccaria Martino, dove tali atti seguono a quelli tradizionali quali le vendite, le donazioni inter vivos o i testamenti. Gli atti di credito si presentano in modo simile dal momento che gli stessi criteri sono presenti ovunque con una sorta di canovaccio comune. Nella maggior parte delle città italiane i notai sono soliti scrivere i contratti di credito su vacchette, che contengono le informazioni principali relative al negozio direttamente davanti agli interessati trascrivendo poi in un secondo momento i medesimi atti in registri nei quali è proposta la versione estesa dei documenti. La scrittura della minuta contiene generalmente in modo sintetico tutte le clausole di garanzia; la successiva registrazione nel protocollo notarile è autentificata dal notaio e garantita dall'autorità pubblica. La pratica dell'uso dei quaderni di minuta è contemporanea all'aumento degli atti di credito e alla loro organizzazione nei formulari notarili. Saranno oggetto di approfondimento tra gli altri il mutuo semplice, il deposito, le vendite a credito, la soccida e le numerose tipologie di prestito dissimulato.

Parole chiave: credito; formulari notarili; Medioevo; Nord Italia; prestatori

* Italian Review of Legal History, 7 (2021), n. 19, pagg. 661-676

* https://riviste.unimi.it/index.php/irlh/index

* ISSN 2464-8914 - DOI 10.54103/2464-8914/16904. Articolo pubblicato sotto Licenza CC-BY. 
Chiunque abbia consultato i registri notarili conservati negli archivi italiani non può non avere rilevato tra gli atti registrati l'alto numero di contratti di obbligazione presenti. Nel corso del XI secolo sono attestate forme di credito che potremmo definire a piccola scala, sovente non registrate dai notai, e, nel caso in cui lo fossero state, non conservate se non in rari casi concernenti prestiti ingenti tra monasteri e casate nobiliari. Le prime testimonianze di atti creditizi custodite negli archivi dell'Italia centro-settentrionale risalgono, infatti, alla fine dell'XI secolo e sono state prodotte nell'area lucchese e in quella bergamasca ${ }^{1}$.

Durante una prima fase collocabile tra la fine dell'XI e l'inizio del XII secolo, i prestiti erano soprattutto su pegno fondiario ${ }^{2}$. Dagli anni intorno al 1180 viene concepito un contratto più semplice, il mutuum, che consentiva di cedere facilmente in prestito piccole somme di denaro la cui restituzione poteva essere anche a breve termine senza la necessità di garantirne il pagamento attraverso l'istituzione di un pegno. La nuova tipologia creditizia si diffuse rapidamente come risulta evidente dalla mole di documenti conservati negli archivi. Un ulteriore semplificazione che agevolò la pratica di registrare i prestiti si deve all'utilizzo del registro, nel quale il notaio redigeva un breve riassunto dell'atto. I primi registri di cui si rimasta traccia nella documentazione sono conservati a Genova e risalgono alla metà del secolo XII, laddove nella maggioranza delle città sono menzionati solamente dai primi anni del Duecento e generalmente conservati dalla seconda metà del secolo ${ }^{3}$.

L'aumento di documenti concernenti obbligazioni nelle fonti è evidente nel corso del XIV secolo quando nei registri notarili è attestata una quantità notevole di atti di natura privata ${ }^{4}$. Va ricordato che un notaio era solito produrre mediamente nel corso della giornata dai tre ai dieci documenti; di conseguenza un registro concernente un arco cronologico di alcuni mesi o di qualche anno può contenere decine se non centinaia di documenti certificanti contratti di obbligazione, che riguardano nella maggior parte dei casi una pluralità di clienti; costoro, infatti, era soliti rivolgersi al professionista della scrittura per avere una documentazione ritenuta valida secondo la legge. Tutte queste transazioni fondate sul credito permettono di conoscere l'entità e la sostanza dell'attività economica locale. L'espansione della documentazione notarile coincide infatti con lo straordinario sviluppo economico del periodo comunale, che poggiava proprio sul credito.

\footnotetext{
1 Bougard, 2010; De Malafosse, 1951; Lewis, 1971; Chévedille, 1974.

2 Sul tema del prestito su pegno fondiario si veda Violante 1962; Id., 1980. L'autore ha messo in luce inoltre la frequenza di atti di donazione ai monasteri contenti la clausola di retrocessione al donatore (ad tenendum et laborandum et fictum faciendum), sorta di contratti di prestito dissimulato con caratteristiche diverse rispetto a quelle attestate comunemente. Si legga anche il lavoro sui prestiti dissimulati in Lombardia: Rossetti, 2008.

3 Bartoli Langeli, 2001.

4 Sulla produzione documentaria nelle città italiane vedi il panorama proposto da Cammarosano, 1991, pp. 125--276.
} 
Va sottolineato che all'interno dei registri notarili sono identificabili unità caratterizzate da comuni destinatari: vi sono infatti notai che operano esclusivamente o quasi per conto di un vescovo piuttosto che di una casata o di un ente assistenziale o addirittura di una comunità di forestieri come ad esempio è attestato nel caso di un notaio attivo nel XV secolo nella città di Treviso: Antonio del fu Cristoforo di Portogruaro si spostò dalla città della Terraferma veneta a Venzone in Friuli per continuare a rogare atti prevalentemente di credito per conto della locale comunità ebraica i cui componenti erano vissuti in precedenza proprio a Treviso. Dopo un periodo di attività nelle terre che furono del Patriarcato di Aquileia il notaio rientrò nella città veneta, dove continuò a lavorare negli anni successivi custodendo quanto prodotto per il nucleo ebraico friulano. Dopo la sua morte tali atti confluirono insieme al resto della documentazione dapprima nell'archivio del collegio dei notai e successivamente nell'archivio di Stato, dove oggi sono conservati ${ }^{5}$.

Gli stessi notai talora decidevano di suddividere gli atti notarili per tipologia nei diversi registri. Piuttosto comune è la scelta di unire nella medesima vacchetta $i$ testamenti così come sembra essere stata abbastanza diffusa la scelta di riunire in uno stesso registro le ipoteche. La maggior parte dei notai sceglieva tuttavia di riunire in modo promiscuo gli atti nel medesimo registro, che di fatto finiva per contenere una varietà di tipologie di contratti con numerosi destinatari. Nella documentazione è molto diffusa la presenza di atti certificanti l'attività di credito dalle forme più semplici riguardanti il prestito semplice di denaro ai contratti di natura squisitamente mercantile, quali ad esempio contratti di cambio o depositi di capitali. Uno dei primi lavori sul rapporto esistente tra la produzione notarile e lo sviluppo creditizio si deve ad un volume pubblicato dall'Ecole Française de Rome e curato da François Menant e da Odile Redon nel 2004 dal titolo Notaires et crédit dans l'Occident méditerranéen médiéval ${ }^{6}$. Gli autori coinvolti nel progetto, dopo aver preso in esame sia la situazione creditizia di vari luoghi e regioni del nord e centro Italia sia le pratiche in uso nella zona mediterranea francese e in Catalogna, giunsero alla conclusione che si potesse parlare di una sorta di età d'oro delle pratiche creditizie e del notariato in un periodo identificato tra la metà del XIII secolo e il 1350.

Come abbiamo ricordato il bisogno di avere a disposizione denaro è antecedente al notariato, che si sviluppò sull'onda di una necessità sociale e politica atta a garantire attraverso la produzione di forme di scrittura documenti ritenuti validi dalla collettività in un periodo caratterizzato da un aumento notevole degli scambi di natura mercantile e commerciale. L'importanza delle transazioni a credito nel quotidiano lavoro dei notai si riflette nell'elaborazione delle formule corrispondenti nei grandi manuali di notariato redatti dai maestri, principalmente bolognesi, durante il Duecento: i vari tipi di mutui e di vendita con pagamento

\footnotetext{
5 Davide, 2004.

${ }^{6}$ Menant, Redon (eds.), 2004.
} 
ritardato furono progressivamente classificati e successivamente inseriti in formulari specifici. Generalmente gli atti di credito trovavano spazio nei formulari dopo i documenti tradizionali quali le vendite, le donazioni inter vivos o i testamenti senza che ne siano specificate in modo coerente le caratteristiche come è possibile constatare nella versione tardiva della produzione irneriana. Con il Liber formularum del 1214 e l'Ars Notarie del 1220 Ranieri di Perugia, incaricato della redazione del Registro Grosso dal comune di Bologna, dove fondò la corporazione dei notai, iniziò a diversificare le formule in uso nei contratti di prestito ${ }^{7}$.

Dopo questa prima classificazione il notaio Salatiele, altro docente dello Studium bolognese, ritornò sulla necessità di organizzare le tipologie di credito nella prima e seconda redazione dell'Ars Notarie prodotta tra il 1253 e il $1254^{8}$. Fu, infine, il celebre Rolandino Passeggeri a decidere di inserire all'interno della Summa artis notarie, un capitolo dedicato esclusivamente al tema dal titolo De debitis et creditis una buona parte del quale è dedicata ai prestiti e alle modalità di rimborso ${ }^{9}$. L'importanza dei rapporti di credito spinse uno degli allievi di Raniero da Perugia, il notaio Zaccaria Martino a decidere di aprire la sua Summa, scritta tra il 1264 e il 1281, con il tema del credito suddiviso in 24 formule, che rispondono alla necessità di avere a disposizione un formulario per fissare i numerosi atti creditizi dei quali era richiesta una redazione, una necessità cresciuta nel corso del tempo in ambiente notarile ${ }^{10}$. Si tratta di un repertorio che potremmo definire come essenzialmente tecnico; Salatiele evitava infatti di affrontare la tematica dell'usura, tema centrale nelle pratiche creditizie $^{11}$. Le artes notariae distinguevano quattro tipologie principali di credito: il prestito semplice, il deposito, il prestito su pegno fondiario e la vendita anticipata del raccolto. L'elaborazione di formulari contenenti un corpus di quelli che si potevano considerare come atti di credito non è limitata alla sola Bologna; ci sono testimonianze analoghe a Padova e a Firenze e fuori d'Italia.

La redazione degli atti di credito nell'Italia centrale e settentrionale era caratterizzata da un'intelaiatura ricorrente in cui sono presenti almeno gli elementi principali del negozio giuridico. Nella maggior parte delle città i notai erano soliti scrivere i contratti di credito su vacchette, che contenevano le informazioni principali relative all'accordo, direttamente davanti agli interessati, nella via pubblica, in casa o nelle botteghe. La stesura della minuta in quaderni di piccolo formato garantiva la possibilità di annotare in modo sintetico i fondamenti del contratto e precedeva il momento della redazione definitiva e la relativa trascrizione nel

7 Gaudenzi (ed.), 1890; Wahrmund (ed.), 1917. Sulla redazione del Registro Grosso si veda Gaudenzi, 1963, pp. 131-168.

8 Orlandelli G. (ed.), 1961.

9 Rolandino Passeggeri, Summa artis notarie, Lyon, 1565; Sinisi, 2002.

10 Ferrara (ed.), 1993.

11 Sul tema dell'usura cfr. Langholm, 1992; Todeschini, 2002; Quaglioni, Todeschini, Varanini (eds.) 2006. 
registro dei protocolli autentificati dal notaio e garantiti dall'autorità pubblica ${ }^{12}$. La pratica dell'uso dei quaderni di minuta è contemporanea all'aumento degli atti di credito e alla loro organizzazione nei formulari notarili. Di fatto nei quaderni di minuta i documenti di natura creditizia - sia quelli riguardanti contratti rogati in città sia quelli registrati nelle campagne- erano numerosi. Per gli attori principali, creditore e debitore, tali atti presentavano le stesse garanzie degli instrumenta originali.

Vediamo ora quali fossero le forme del credito più in uso nelle città e nelle campagne del Nord d'Italia ${ }^{13}$. Caratterizzate in genere dalla sottolineatura della restituzione piuttosto che dell'investimento, il prestito al consumo e le vendite a credito divennero estremamente diffuse in tutti i ceti sociali. I mutui furono fondamentali per lo sviluppo dell'artigianato e dei commerci delle città e divennero necessari per sostenere il lavoro dei contadini, che non erano sempre in grado di accumulare le scorte di cui necessitavano. Le somme di denaro loro concesse erano in genere modeste e dipendevano dal calendario delle attività agricole come è evidente anche nei termini di restituzione frequentemente fissati nelle tradizionali feste di San Michele del 29 settembre e di San Martino dell'11 novembre $^{14}$. Le due tipologie di credito più frequenti tra il XIII e il XV secolo nella documentazione dell'Italia settentrionale sono il mutuo semplice, definito come purum mutuum, instrumentum o carta debiti, e il deposito, chiamato nelle fonti quale instrumentum o carta depositi o salvamenti. Nel primo caso si trattava del documento con cui si registrava la cessione di denaro a prestito; dopo aver indicato l'entità della somma concessa i notai inserivano i tempi di restituzione, che nel tardo Medioevo erano sovente a breve se non brevissimo termine, ed istituivano le penalità in caso di insolvenza. Non era sempre indicato invece il tasso di interesse percepito sulla somma concessa una volta concluso l'iter dell'operazione; molto probabilmente il debito indicato nei documenti era in realtà maggiore di quello reale e includeva anche l'utile. Non è sempre chiaro, invece, se il debitore pagasse un interesse superiore a quello previsto nella normativa statutaria dei Comuni italiani, in genere calcolato intorno al $10 \%$ della somma prestata, e pertanto usurario. Va ricordato che dalla fine del XIII secolo le città iniziarono ovunque ad adottare l'interdizione completa del prestito a interesse promulgata dai due concili di Lione del 1245 e del 1274 e da quello di Vienne del 1311. In molte zone del Nord d'Italia verso la metà del Trecento il saggio d'interesse iniziò

\footnotetext{
12 II privilegio che garantiva la publica fides agli atti rogati dai notai era ritenuto un requisito indispensabile già nel XIII secolo come evidenziava Rainero di Perugia nel Liber formularum in particolare nel passaggio «aliquando a domino papa, vel imperatore, vel spectabilibus regie curie viris, seu comitibus palatinis, aut alias a principe iurisdictionem habentibus, aliquando a rectoribus civitatum postulantibus erogatur»: Gaudenzi, 1962, pars secunda, rubr. XXXVIIII de officio notarie, indi rubr. XXXX-XLIV, pp. 25-67, a p. 64.

13 Sulle tipologie di credito in uso nel Nord d'Italia si veda Davide, 2021.

${ }^{14}$ Sul credito rurale si veda Gaulin, Menant, 1998; Menant, 2004a.
} 
a non essere più indicato e fu sostituito da una sorta di generica motivazione del perché fosse stato acceso il mutuo, indicato con formule molto semplici quali recepisse ad mutuum, nomine mutui, causa mutui boni amoris et dilectionis, pro bono et fino amore, de vero sorte et iusto capitali, gratis et amore, bona et mera fede. L'uso di tali formule aumentò in alcune zone, ad esempio in Friuli e nel Veneto, e specificatamente a Treviso e nel Bellunese, molto probabilmente in seguito ai provvedimenti specifici assunti dalle autorità negli anni Quaranta del secolo contemporaneamente all'emanazione delle costituzioni sinodali contro I'usura da parte del patriarca di Aquileia ${ }^{15}$.

Va ricordato che l'interesse imposto sul prestito, sovente incluso senza essere dichiarato nella somma di denaro da rimborsare, poteva aumentare nel caso di pagamenti effettuati in ritardo sui quali erano previsti ulteriori rincari supplementari. La tendenza a tacere il tasso d'interesse si diffuse in Lombardia in precedenza rispetto a quanto attestato nell'Italia nord-orientale; le prime testimonianze risalgono alla metà del XIII secolo e anche in questo caso rappresentarono l'esito di provvedimenti contro la diffusione dell'usura emanati dalla Chiesa che avrebbero comportato l'abrogazione delle norme sui tassi di interesse in varie legislazioni comunali tra le quali si ricordano quelle di Vercelli e di Ivrea ${ }^{16}$. Nel caso di Novara in particolare si assiste alla cancellazione di tutte le norme relative all'interesse percepito sui mutui con l'inserimento di una norma in cui si affermava che il concetto di usura era lo stesso indicato nel diritto romano ${ }^{17}$. È evidente l'influenza della predicazione degli Ordini Mendicanti sulle decisioni che hanno comportato un cambiamento nella normativa statutaria relativa ai tassi di interesse, alle tutele dei creditori nei confronti dei debitori e al prestito su pegno ${ }^{18}$.

Nella documentazione sono attestati anche numerosi contratti di deposito, sebbene in quantità inferiore rispetto agli atti di prestito; la prassi prevedeva che gli investimenti fossero fatti in genere presso prestatori o cambiavalute, che non necessariamente esercitavano anche la professione feneratizia. In questa tipologia di contratti era spesso omessa l'indicazione dei tempi di restituzione mentre era sempre riportata la promessa di resa al depositante della somma di denaro ricevuta come deposito qualora lo avesse richiesto. Per quanto riguarda invece la vendita anticipata di beni agricoli, quali ad esempio grano e olio, il venditore era solitamente identificabile nel contadino, che aveva prodotto tali beni e che

\footnotetext{
15 Si tratta delle costituzioni sinodali contro l'usura volute dal patriarca di Aquileia e del vescovo di Treviso, Pietro Paolo Costa del XIV secolo. Sulle tipologie creditizie in uso in Veneto nel Medioevo cfr. Varanini, 1983; Caliaro, 1983; Cagnin, 2004, pp. 97-124. Sul credito nel Patriarcato di Aquileia si veda Davide, 2003, 2019, 2020.

16 Mainoni, 2005, pp. 147-151; Vauchez, 1990, pp.119-159; Baietto, 2002, pp. 518-519.

17 Norma 74 degli Statuta Communitatis Novariae, ed. A. Cerruti, Torino, Novariae, Ex typographeo fratrum Miglio 1878, p. 579 (Historia Patriae Monumenta, XVI, Leges I).

18 Sulle modalità di accesso al credito su pegno da parte delle istituzioni ecclesiastiche si veda Pigozzo, 2012.
} 
si trovava a non avere più il necessario per sfamare la famiglia fino al raccolto successivo. La cessione equivaleva ad una sorta di prestito, attestato sempre alla fine d'inverno o all'inizio della primavera, in cui non erano contemplati interessi. II guadagno del prestatore era comunque considerevole poiché in genere acquistava le merci per un prezzo basso.

Nella parte finale della maggior parte dei contratti di prestito e di deposito erano inserite le sanzioni a carico della parte inadempiente. Già dalla metà circa del XIII secolo sono testimoniate le prime attestazioni concernenti le penalità previste per il debitore insolvente, calcolate nell'equivalente del doppio della somma di denaro avuta in prestito. II creditore era solito tutelarsi richiedendo al debitore una garanzia reale sui propri beni anche in questo caso pari al doppio del debito. Alla persona morosa, inoltre, era sovente richiesta l'indicazione di un fideiusso$\mathrm{re}$, che garantiva il pagamento nel caso di insolvenza. Nella documentazione del Nord d'Italia è inserita spesso la formula con cui il debitore rinunciava ad ogni forma di tutela legale e si impegnava a osservare la validità e, se vogliamo, anche l'integrità fisica dell'atto certificante il debito fino al momento della restituzione di quanto avuto in prestito. In Lombardia e in Veneto si inseriva la richiesta di mostrare l'atto di quietanza come garanzia dell'avvenuto pagamento del mutuo e spesso era presente la rinuncia a quanto disciplinato dagli statuti cittadini a difesa degli interessi dei debitori nei contratti di mutuo concernenti più persone in solidum; in tal modo al creditore era accordata la possibilità di agire contro l'uno o l'altro dei debitori per avere indietro l'intera somma prestata e non solamente la quota spettante a ciascuna parte in causa. Nel Patriarcato di Aquileia e in Veneto, molte di queste carte di debito sono seguite da un atto notarile indicato come promissio conservationis indemnitatis, in cui il debitore principale liberava gli altri dall'obbligo nei riguardi del creditore. Si trattava in sostanza di garantire le persone che avevano solidalmente acceso il mutuo relegandole ad un ruolo di puri garanti.

Le procedure relative ai prestiti prevedevano come atto finale la stesura da parte di un notaio di una carta finis, ovvero di una quietanza nella quale si attestava l'avvenuta restituzione di un prestito o di un deposito. Solitamente, una volta ricevuta la restituzione, il creditore consegnava al debitore il relativo instrumentum cancellato tramite due tratti in diagonale o inciso. I debitori difficilmente riuscivano a rispettare i tempi previsti per la restituzione dei prestiti e di conseguenza i rimborsi venivano fatti in ritardo: la prassi prevedeva la rogazione di ulteriori atti nei quali erano registrati gli interessi suppletivi. I notai non si limitavano alla redazione di queste tipologie di documenti giocando un ruolo centrale in tutte le cause giudiziarie promosse contro i debitori insolventi come componenti degli uffici del comune. Un valido esempio è quello del registro dei bandi per debiti di Bologna in cui i notai registravano i debiti non pagati e l'eventuale cancellazione una volta risarciti ${ }^{19}$.

${ }_{19}$ Sui registri dei bandi per debiti di Bologna si veda Gaulin, 1997. Sulla disciplina del 
La tipologia creditizia più diffusa nel XIV e XV secolo, insieme al prestito al consumo, fu la vendita a credito di generi alimentari, soprattutto cereali, ma anche di animali e di panni presente in numerosi esemplari nella maggior parte dei registri notarili del nord d'Italia. Evidentemente le cessioni a credito di beni agricoli, quali le granaglie e il vino, avevano spesso un carattere speculativo essendo del tutto influenzate dal calendario agricolo. Nel corso del Trecento tali vendite aumentarono e di regola si diffuse l'abitudine di garantire il pagamento attraverso l'istituzione di guarentigie, costituite in genere da animali o beni immobili. Non erano soli i ceti rurali ad acquistare a credito i cereali dal momento che erano soliti farlo anche gli abitanti dei comuni, tra i quali spiccavano molti mercanti e piccoli artigiani abituati a praticare a latere delle loro principali attività il prestito ad interesse.

Oltre a queste forme contrattuali di vendite a credito e di prestito si ritrovano nella documentazione altre tipologie creditizie le più note delle quali sono le vendite fittizie, frequenti soprattutto in Lombardia e in Veneto. Attestate già nel corso del XIII secolo, con un formulario del tutto simile a quello usato nel periodo successivo, mascheravano un prestito che veniva garantito attraverso il trasferimento al creditore di beni immobili, che sarebbero stati poi eventualmente restituiti al debitore attraverso un contratto di recreditio o retrovendita al debitore una volta restituito il prestito. Sono inoltre presenti nelle fonti anche esempi di contratti di credito nei quali era dato un valore monetario ai canoni d'affitto riscossi in natura per poter consentire di stabilire una sorta di tasso d'interesse, oltre a numerosi atti di prestito a terzi attraverso atti regolari di vendita e contratti certificanti il trasferimento di beni seguiti da prestiti.

Tra le diverse forme di credito all'agricoltura si segnala il contratto di affidamento degli animali declinato in numerose tipologie di contratti ${ }^{20}$. La più diffusa prevedeva la soccida con una divisione al $50 \%$ del valore dell'animale e dei prodotti ottenuti in un periodo di custodia determinato. Di solito la spesa della sorveglianza e del mantenimento degli animali gravava sulla sola figura del soccidario. Nel Patriarcato di Aquileia i contratti includevano nella maggior parte dei casi la possibilità di morte dell'animale; qualora il decesso non fosse dovuto alla cattiva condotta del soccidario il danno calcolato doveva essere risarcito solamente a metà. Nel caso di buoi o vacche i tempi di nolo avevano speso un termine fissato non oltre i due anni di tempo. Negli accordi era garantita al proprietario degli animali la possibilità di controllare il modo con cui erano custoditi e alimentati. Nelle zone in cui era diffusa questa clausola, come ad esempio nel Friuli Occidentale, nel caso eventuale di morte o ferimento dell'animale coloro che lo avevano preso a noleggio erano tenuti a risarcire il proprietario di tutti i danni stimati da un'apposita commissione composta dagli uomini di fiducia del Comune ${ }^{21}$.

bando in città cfr. Milani, 1997.

20 Sulla soccida cfr. Cortonesi, 2006.

21 Davide, 2020, pp. 706-707. 
Nelle zone marginali delle campagne friulana e veneta la maggior parte dei contratti di soccida era stipulata durate i mesi autunnali. Di durata spesso quinquennale le soccide erano utilizzate come una sorta di integrazione nei contratti di locazione di terreni e prati e non si possono considerare solo come atti certificanti l'affitto di animali. Una tipologia di contratto diffusa nella documentazione veneta, friulana e lombarda univa inoltre la soccida ai contratti di deposito: si trattava di un atto notarile definito nelle fonti come carta depositi di un animale; tale atto era redatto senza che fossero indicati sia la durata del contratto sia il tempo di restituzione, i quali erano lasciati all'arbitrio del proprietario. Tale modalità di credito all'agricoltura coinvolgeva proprietari e contadini ma anche molte persone estranee al mondo agricolo, spesso mercanti cittadini, interessate agli ampi margini di guadagno che potevano conseguire. Va sottolineato che questo accordo non aveva sempre una natura usuraia e spesso rispondeva alle esigenze degli abitanti delle città, che avevano bisogno di generi alimentari e materie prime. L'investimento oltre a garantire un guadagno poteva inoltre rappresentare un volano per particolari ambiti di attività come è evidente nel caso dei numerosi contratti di soccida stipulati a Pavia nel corso del XIII e XIV secolo dagli asinari22.

Di fronte alla varietà degli atti certificanti transazioni creditizie si palesa la costante ed evidete difficoltà incontrata dai notai nello scrivere chiaramente il tasso d'interesse soggetto ai divieti contro l'usura. I contratti per essere considerati legali non potevano dichiarare un interesse diverso da quello permesso dalla Chiesa e previsto nella legislazione vigente. Se, come abbiamo ricordato, i notai evitavano comunemente di registrare il tasso nei contratti di vendita fittizia, il problema si faceva più evidente nei mutui nei quali la soluzione più diffusa fu quella di omettere il tasso d'interesse richiesto limitandosi ad indicare solamente la somma da rimborsare. Un'altra soluzione adottata, in particolare dai prestatori professionisti, quali furono i lombardi e i toscani, fu sovente quella di giocare con le diverse coniazioni formulando il prestito come se fosse un contratto di cambio.

Nella documentazione sono attestati inoltre, anche se in misura largamente minore, prestiti garantiti attraverso forme di scrittura chirografaria. Si trattava di un espediente usato da coloro che volevano evitare il ricorso ai notai e la cui esistenza è documentata in modo indiretto essendo citata nelle cause giudiziarie, negli atti di ultima volontà e negli inventari, fonti nelle quali spesso si ritrovano note $\mathrm{e}$ informazioni sul panorama delle attività creditizie praticate in un territorio. Nei testamenti, in particolare, si possono ritrovare elenchi dettagliati dei debiti e dei crediti del defunto con le relative annotazioni su pegni ceduti o avuti in garanzia. In questi casi solitamente era segnalata la data di redazione dell'atto creditizio e l'ammontare del debito e del credito. Si tratta pertanto di una fonte ricca di informazioni sugli investimenti fatti da persone, che sovente non hanno lasciato altre tracce della loro attività nella documentazione. L'analisi puntale dei rapporti di debito-credito presenti nei testamenti e negli inventari friulani ha dimostrato

22 Bertoni, 2013, pp. 159-160. 
come fosse comune per una persona rivestire più ruoli nel corso del tempo: il debitore poteva diventare creditore e successivamente testimone di vendite a credito o di prestiti. Anche nelle realtà minori si era costituita una rete creditizia articolata come suggeriscono le dichiarazioni di alcuni prestatori, che sostengono di aver esercitato l'usura sia a vantaggio personale sia per conto di terzi.

Gli stessi notai, che rogavano una grande quantità di atti di credito, finivano per acquisire le competenze dei prestatori e decidevano di far fruttare il denaro acquisito durante l'esercizio della professione investendolo nel mercato dei prestiti. Si trattava di una scelta esercitata in modo continuato e non saltuario. In particolare sembrano essere stati i notai che vivevano nelle zone rurali a praticare il prestito a fianco della loro attività principale come erano soliti fare anche gli esponenti della classe artigianale. Nella documentazione si ritrovano frequentemente testimonianze di questa attività nelle vacchette dove i notai annotavano $\mathrm{i}$ prestiti che erano soliti concedere. Un ottimo esempio è dato da un quadernetto rogato dal notaio Giorgio di Codroipo, oggi conservato tra le carte dell'archivio di Stato di Udine, nel quale sono registrati tutti i prestiti concessi ${ }^{23}$. Nell'elenco accanto al nome del debitore è fornita l'indicazione del luogo di provenienza: il notaio era solito prestare ad una clientela residente in una zona diversa rispetto a quella in cui vivevano le persone che si affidavano alle sue competenze per la redazione dei documenti. Tale scelta sembra essere stata piuttosto comune tra i notai ed è riscontrata in altre zone dell'Italia settentrionale. In questo tipo di quaderni i debiti vengono sovente divisi tra quelli più recenti e quelli più antichi, spesso oggetto di rivalutazione e con scadenze di restituzione dilatate nel tempo. $\mathrm{Nel}$ caso del notaio a cui ho accennato la lista dei debitori testimonia sia il comune bisogno di un prestito al consumo concernente piccole somme di denaro sia la costante impossibilità di risarcire il debito nei tempi previsti.

Prestatori che provengono dal ceto notarile sono attestati ovunque nel centro e nord d'Italia. Tra i casi più noti si segnala quello dei notai di Bergamo i quali, tra la metà e la fine del Duecento, si specializzarono nel fornire prestiti sempli$\mathrm{ci}$, che erano soliti differenziare in base alla clientela. In uno studio pubblicato da Paolo Gabriele Nobili, giovane collega morto prematuramente alcuni anni fa, sono ricostruite le vicende di una famiglia di Bergamo, i Bonelli, i cui rappresentanti sono contemporaneamente notai e prestatori. I contratti di prestito stipulati dai componenti della famiglia erano diversificati in base ai richiedenti: somme modeste erano concesse alle persone che si erano da poco inurbate in città e che provenivano dalle montagne e dalle colline circostanti mentre erano date in prestito cospicue somme di denaro ad altri notai, inseriti pienamente nei meccanismi dell'economia cittadina. I notai prestatori non concedevano solo prestiti ad interesse ma erano attivi anche nel settore delle vendite a credito in particolare

\footnotetext{
${ }^{23}$ Archivio di Stato di Udine, Notarile Antico, b. 1764, r. 2, c. 2rv. Sull'attività del notaio cfr. Davide, 2020, pp. 698-307.
} 
di granaglie ${ }^{24}$. Nel caso lombardo, oggetto di studio in un noto articolo sul credito rurale a cura di Jean-Louis Gaulin e François Menant dal titolo Crédit rural et endettement paysan dans l'Italie communale, in Endettement paysan et crédit rural dans l'Europe médiévale et moderne, è attestata quasi ovunque la presenza di una rete di piccoli prestatori di villaggio, che cedevano somme di denaro contenute a persone residenti nei dintorni dei luoghi in cui vivevano. I mutui erano registrati soprattutto nella documentazione dei notai che operavano nelle campagne. La struttura del credito in ambito rurale rimanda ad un'immagine composita, che è evidente allorché si prendano in esame e si confrontino le liste dei debitori e quelle dei prestatori, di cui si è conservata traccia nella documentazione. Nel caso lombardo, ad esempio, esisteva una sorta di primo circuito del credito tra prestatori e debitori di villaggio, che potevano essere sia familiari sia compaesani caratterizzati dalla condivisione di un medesimo livello socioeconomico, con rapporti che erano essenzialmente fondati su una certa reciprocità ovviamente non scevra completamente da interessi. Un secondo circuito era quello costituito dai rapporti di credito tra gli abitanti di villaggio, i vicini e i prestatori di professione, dove i legami finivano per essere squilibrati a favore di questi ultimi. Esisteva infine un ultimo livello dove le somme in gioco erano particolarmente elevate: $i$ prestatori coinvolti concedevano ingenti somme di denaro e reinvestivano i proventi nel mercato del credito cittadino. Una simile organizzazione creditizia era presente anche nel Patriarcato di Aquileia: nei centri di medie dimensioni, quali potevano essere Spilimbergo e San Vito al Tagliamento, esistevano infatti diversi circuiti di prestito rispondenti alle molteplici necessità di coloro che avevano bisogno di un supporto economico. Se erano in molti a sentire l'esigenza di un prestito al consumo con piccole somme di denaro da restituire in tempi brevi non mancavano neppure anche coloro che avevano bisogno invece di sostegni finanziari più strutturati come gli esponenti del ceto artigianale e mercantile e i membri della nobiltà locale ${ }^{25}$. Nelle realtà maggiormente sviluppate dal punto di vista economico le transazioni creditizie rispondevano comunque a tutte le esigenze del mercato come è evidente in Piemonte, dove i ben conosciuti prestatori provenienti dalle città di Asti e di Chieri, noti in tutte le principali piazze commerciali europee come "Lombardi", erano soliti investire i cospicui capitali accumulati con l'attività di prestito in Europa nel mercato del credito locale oltre che nell'acquisto di terreni e proprietà ${ }^{26}$. Va peraltro sottolineato che le possibilità offerte dal credito cittadino rappresentavano per i prestatori un mezzo per arrivare ai vertici della società come nel caso dei Beccaria o dei Folperti a Pavia ${ }^{27}$.

Come già ricordato i notai si specializzavano nel rogare gli atti per un determi-

${ }^{24}$ Nobili, 2012, pp. 75-76. Sul credito a Bergamo e più in generale in Lombardia si veda Nobili, 2010; Menant, 1993; Menant, 2004b; Del Bo 2010.

25 Davide, 2011.

${ }^{26}$ Olivieri, 2004; Bordone, 1994, 2003; Fiore, 2015; Scarcia, 2004.

27 Rao, 2018, pp.50-53. 
nato tipo di clientela, abitudine confermata anche in Friuli dove i professionisti offrivano i loro servigi solamente a certi operatori attivi in città. Nel caso di San Vito al Tagliamento è evidente la scelta effettuata da un paio di notai di rogare quasi esclusivamente gli atti, prevalentemente ma non esclusivamente di credito, per conto degli esponenti di un circuito finanziario e mercantile gestito completamente da persone di origine tedesca, che in qualità di osti fornivano alloggio e assistenza ai conterranei. I notai che si specializzavano nel fornire sostegno al mercato del credito, tuttavia, non erano tuttavia impegnati solamente in questo settore dal momento che erano soliti rogare una larga varietà di atti ${ }^{28}$. Come ha sottolineato più volte François Menant nei suoi lavori il credito rivestì un importanza centrale nel lavoro dei notai dell'epoca comunale, e il sistema creditizio dei secoli XII e XIII si fondò sostanzialmente sulla fiducia che creditori e debitori riponevano nelle loro competenze tecniche e conoscenze.

\section{Bibliografia}

Baietto L., 2002: Vescovi e comuni: l'influenza della politica pontificia nella prima metà del secolo XIII a Ivrea e Vercelli, in "Bollettino Storico-Bibliografico Subalpino", C/2, pp. 459-546

Bartoli Langeli A., 2001: /l notariato, in G. Ortalli, D. Puncuh (eds.), Genova, Venezia, il Levante nei secoli XII-XIV. Atti del Convegno internazionale di studi (Genova-Venezia, 10-14 marzo 2000), Genova, Società ligure di storia patria ("Atti della Società ligure di storia patria», n.s., 41/1), pp. 73-101

Bertoni L. 2013: da Pavia alla fine del Duecento. Una società urbana fra crescita e crisi, Bologna CLUEB (Itinerari medievali per la didattica)

Bordone R., 1994: I Lombardi in Europa. Primi risultati e prospettive di ricerca, in "Società e storia", 17/1194, 63, pp. 1-17

Bordone R., 2003, L'uomo del banco dei pegni. Lombardi e mercato del denaro nell'Europa medievale, Torino, Scriptorium, 1994 (ried. Asti, Centro studi sui Lombardi)

Bougard F., 2010: Le crédit dans l'Occident du Haut Moyen Âge, in Les élites et la richesse au Haut Moyen Âge, in J. P. Devroey, L. Feller e R. Le Jan (ed.), Turnhout, Armand Colin, pp. 439-478 (Collection Haut Moyen Âge, dir. R. Le Jan, 10)

Chédeville A., 1974: Le rôle de la monnaie et du crédit dans les pays de l'Ouest de France, XIe-XIIle siècle, in "Cahiers de Civilisation Médiévale", 17 (1974), pp. 305-325

Cagnin G., 2004: "Pro bono et fino amore, de iusto et vero capitali et vera sorte". Documentazione notarile e credito a Treviso (secoli XIII-XIV), in F. Menant, O.

28 Davide, 2011. 
Redon (eds.), Notaires et crédit dans l'occident méditerranéen médiéval, Roma, École Française de Rome, pp. 97-124 (Collection de l' École Française de Rome, 343)

Caliaro E., 1983: II prestito ad interesse a Vicenza tra XII e XIII secolo (1184-1222), in "Studi Storici Luigi Simeoni", 33, pp. 103-120

Cammarosano P., 1991: Italia medievale. Struttura e geografia delle fonti scritte, Roma, Carocci

Cortonesi A., 2006: Soccide e altri affidamenti di bestiame nell'Italia medievale, in A. Cortonesi, M. Montanari, A. Nelli (eds.), Contratti agrari e rapporti di lavoro nell'Europa medievale. Atti del Convegno Internazionale di Studi. Montalcino, 20-22 settembre 2001, Bologna, CLUEB, pp. 203-223

Davide M., 2003: /l credito in Friuli nel Trecento, in "Studi medievali", 3a Ser., XLIV (II), pp. 639-668

Davide M., 2004: La comunità ebraica nella Venzone del Quattrocento, in "Ce fastu?", LXXX (2004), 2, pp. 167-186

Davide M., 2011: Economia e credito a San Vito nel tardo Medioevo e nella prima età moderna, in P.C. Begotti e P.G. Sclippa, San Vît: 87n Congrès, San Vît, ai 3 di Otubar dal 2010, Udine, Società Filologica Friulana, 561-576

Davide M., 2019: Il prestito al consumo e le pratiche creditizie nel Friuli Occidentale del Tardo Medioevo, in A. Fadelli, A. Marcon Andrea (eds.), Documenta artium fundamentum. Studi offerti a Paolo Goi per i suoi ottant'anni, Pordenone, Accademia San Marco, pp. 33-43

Davide M., 2020: Problemi di storia economica in età patriarcale. Le pratiche creditizie nel Friuli Tardo Medievale, in P. Goi, A. Marcon (eds.), Atti dell'Accademia di San Marco. Vol. 29, Pordenone, Accademia San Marco, pp. 689-723

Davide M., 2021: Gli operatori del credito e le pratiche in uso nel Nord d'Italia tra XIV secolo e XV secolo /Credit operators and practice in use in Northern Italy between 14th and 15th centuries, in II credito. Fiducia, solidarietà, cittadinanza (secc. XIV-XIX)/The credit. Trust, solidarity and citizenship(14th-19th centuries), RiMe 8/II n.s. (June 2021). Special Issue, pp.45-69

Del Bo B., 2010: Banca e politica a Milano a metà Quattrocento, Roma, Viella

De Malafosse J., 1951: Contribution a l'étude du credit dans le Midi aux X e XI siècles: le sûretés réelles, in "Annales du Midi", 63 (1951), pp. 105-148

Ferrara R. (ed.), 1993: Summa artis notarie di Martino Zaccaria, Bologna, ISTUB (Opere dei Maestri)

Fiore A., 2015: Dal prestito al feudo. Percorsi di affermazione signorile nel Piemonte meridionale del Trecento, in "Bollettino Storico-Bibliografico Subalpino", anno CXIII/2015, fasc. I, gennaio-giugno, pp. 189-226 
Gaudenzi A. (ed.), 1890: Rainerii de Perusio Ars Notaria, Bologna, In Aedibus Societatis Azzoguidiane

Gaudenzi A. (ed.), 1962: Ranieri da Perugia, Ars notaria, in Bibliotheca luridica Medii Aevi (Scripta anecdota antiquissimorum glossatorum), vol. II, Bononiae, in aedibus Petri Virano olim fratrum Treves, 1892, rist. anast. Torino, Bottega d'Erasmo

Gaulin J.L., Menant F., 1998: Crédit rural et endettement paysan dans l'Italie communale, in J.L. Gaulin, F. Menant (eds.), Endettement paysan et crédit rural dans l'Europe médiévale et moderne, Toulouse, Presses Universitaires du Mirail, pp. 35-67

Gaulin J.L., 1997: Les registres de bannis pour dettes à Bologne au XIIle siècle: une nouvelle source pour l'histoire de l'endettement, in "Mélanges de l'école française de Rome", 1997, 109-2, pp. 479-499

Langholm O., 1992: Economics in the Medieval Schools. Wealth, Exchange, Value, Money and Usury according to the Paris Theological Tradition, Leiden, Brill

Lewis P.A., 1979: Mortgages in the Bordelais and Bazadais, in "Viator. Medieval and Renaissance Studies, 10 (1979), pp. 23-38

Mainoni P., 2005: Credito e usura fra norma e prassi. Alcuni esempi lombardi (sec. XII-prima metà XIV), in D. Quaglioni, G. Todeschini, G.M. Varanini (eds.), Credito e usura fra teologia, diritto e amministrazione. Linguaggi a confronto (sec. XII-XVI), Roma, École française de Rome, pp.129-158 (Collection de l' École française de Rome, 346)

Menant F., 1993: Campagnes lombardes du Moyen Âge. L'economie et la société rurale dans la région de Bergame, de Crémone et de Brescia du Xe au XIIle siècle, École Française de Rome, Roma (Bibliothèque Des Ecoles Françaises d' Athènes Et De Rome, Fasc. 281)

Menant F., Redon O. (eds.), 2004: Crédit et notaires dans l'Occident méditerranéen médiéval, Roma, École française de Rome (Collection de l'École française de Rome, 343)

Menant F., 2004a: Pour une histoire de l'information sur le crédit rural au Moyen Âge. Esquisse de problématique et études de cas in Italie du Nord au XIle-XIVe siécles, in C. Boudreau, K. Fianu, C. Gauvard, M. Hébert (eds.), Information et société en Occident à la fin du Moyen Âge. Paris, Publications de la Sorbonne, pp. 135-150 (Histoire ancienne et médiévale, 78)

Menant F., 2004b: Notaires et crédit à Bergame à l'epoque communale, in F. Menant, O. Redon (eds.), Crédit et notaires dans l'Occident méditerranéen médiéval, Roma, École française de Rome (Collection de l'École française de Rome, 343), pp. 31-54

Milani G., 1997: Prime note su disciplina e pratica del bando a Bologna attorno alla metà del XIII secolo. II bando e i suo usi, in "Mélanges de l'école française 
de Rome", 1997, 109-2, pp.501-523

Nobili P.G., 2010: Alle origini della fiscalità comunale. Fodro, estimo e prestiti a Bergamo fra fine XII e metà XIII secolo, in "Reti Medievali - Rivista", 11, 1, pp. 1-78

Nobili P.G., 2012: Alle origini della città. Credito, fisco e società nella Bergamo del Duecento, Bergamo, Fondazione per la storia economica e sociale di Bergamo (Collana Studi di storia della società, dell'economia e delle istituzioni bergamasche, 5)

Olivieri A., 2004: La documentazione delle operazioni creditizie nell'archivio di una famiglia vercellese (secolo XIV), in F. Menant, O. Redon (eds.), Crédit et notaires dans l'Occident méditerranéen médiéval, Roma, École française de Rome (Collection de l'École française de Rome, 343), pp. 97-124

Orlandelli G. (ed), 1961: Salatiele, Ars notarie, Milano, ISTUB (Istituto per la storia dell'Università di Bologna, Opere dei maestri, 2)

Orlandelli G., 1963: Il sindacato del podestà: la scrittura da cartulario di Ranieri da Perugia e la tradizione tabellionale bolognese del 12 secolo, Bologna, R.Patron;

Pigozzo F., 2012: La pratica del prestito su pegno e le istituzioni ecclesiastiche nel tardo medioevo, in M. Carboni e M.G. Muzzarelli, In Pegno. Oggetti in transito tra valore d'uso e valore di scambio (secoli XIII-XX), Bologna, II Mulino, pp. 115131

Quaglioni D., Todeschini G. e Varanini G.M. (eds.), 2006: Credito e usura fra teologia, diritto e amministrazione. Linguaggi a confronto (sec. XII-XVI), Roma, École française de Rome (Collection de l' École française de Rome, 346)

Rossetti G., 2008: Motivi economico-sociali e religiosi in atti di cessione di beni a chiese del territorio milanese nei secoli XI e XII, in Percorsi di Chiesa nella società medioevale. Il culto dei santi, il patrimonio, i vescovi, il clero, le donne, le voci del tempo, un papa riformatore, un epilogo, Pisa, Gisem-Edizioni ETS, pp. 151214 (Piccola Biblioteca Gisem, 25)

Rao R., 2018: Credito, diversificazione, integrazione regionale e mercato locale a Pavia prima della Peste (1290-1361), in B. Figliuolo (ed.), Centri di produzione, scambio e distribuzione nell'Italia centro-settentrionale. Secoli XIII-XIV, Udine, Forum, pp. 41-76;

Scarcia G., 2004: La typologie des actes de crédit: les mutua des "Lombards" dans le registres notariés du XIVe siècle, in F. Menant, O. Redon (eds.), Crédit et notaires dans l'Occident méditerranéen médiéval, Roma, École française de Rome (Collection de l'École française de Rome, 343), pp. 149-171

Sinisi G., 2002: Alle origini del notariato latino: la Summa Rolandina come modello di formulario notarile, in G. Tamba (ed.), Rolandino e l'Ars Notaria da Bologna all'Europa. Atti del convegno internazionale di studi storici sulla figura e l'opera di Rolandino (Bologna, 9-10 ottobre 2000), Milano, Giuffrè, pp. 163-234 
Todeschini G., 2002: I mercanti e il tempio. La società cristiana e il circolo virtuoso della ricchezza fra Medioevo e Età Moderna, Bologna, II Mulino

Varanini G.M., 1983: Tra fisco e credito: note sulle camere dei pegni nelle città venete del Quattrocento, in L'attività di prestito nella Repubblica veneta e negli antichi Stati italiani, Studi storici Luigi Simeoni, 33, pp. 215-246

Vauchez A., 1990: Ordini mendicanti e società italiana (XII-XV secolo), Milano, II Saggiatore

Violante C., 1962: Les prêts sur gage foncier dans la vie économique et sociale de Milan au Xle siècle, in "Cahiers de civilisation médiévale", V (1962), pp. 147$168,437-459$

Violante C., 1980: Monasteri e canoniche nello sviluppo dell'economia monetaria (secoli XI-XIII), in Istituzioni monastiche e istituzioni canonicali in Occidente (1123-1215). Atti della settima settimana internazionale di studio, Passo Mendola, 28 agosto-3 settembre 1977, Milano, Vita e Consiglio. Pubblicazioni dell'Università Cattolica del Sacro Cuore

Wahrmund (ed.), 1917: Die Ars Notariae des Rainerius Perusinus, in Quellen zur Gesch. des römisch-kanonischen Processes im Mittelalter, vol. III, 2, Innsbruck, Wagner 